\title{
Assessing Caregiver Burden in Caregivers of Patients with Schizophrenia and Bipolar Affective Disorder in Kathmandu Medical College
}

\author{
Rachana Sharma, ${ }^{1}$ Subhash Chandra Sharma, ${ }^{1}$ Sudarshan N Pradhan ${ }^{1}$ \\ ${ }^{1}$ Department of Psychiatry, Kathmandu Medical College Sinamangal, Kathmandu, Nepal.
}

\begin{abstract}
Background: Schizophrenia and bipolar affective disorder are chronic psychiatric illness that requires long term care. This study tends to measure psychological burden and factors associated with it among caregivers of these two illnesses.

Methods: This is a cross- sectional study that included participants by purposive sampling method. Self designed performa was used to collect the socio-demographic details of the caregivers. Modified caregiver strain index was used to assess the overall stress. Beck's depression inventory and Beck's anxiety inventory was used to assess depression and anxiety respectively.

Results: Hundred caregivers, 50 each of schizophrenia and bipolar affective disorder were enrolled. Seventy-two percent of caregivers were found to have higher level of stress. Twenty-five percent had depression and 29\% anxiety related problems. Stress was found to be significantly associated with being in debt, longer duration of illness, education level, marital status, subjective feeling of psychological stress and self- acknowledgement of need of professional help. Caregivers of both group experienced similar level of stress.

Conclusions: Psychological burden is seen to be high in caregivers of patients of Schizophrenia and Bipolar Affective Disorder.

Keywords: Bipolar affective disorder; caregiver; schizophrenia; stress.
\end{abstract}

\section{INTRODUCTION}

Care-giving is important for both physical and mental disorders in chronic and disabling conditions. The factors like, spending extra hours, financial burden, less time to care for themselves have been associated with high level of stress leading to deterioration of physical, psychological and social health. ${ }^{1-4}$

Schizophrenia and Bipolar Affective Disorder (BPAD) are among the chronic psychiatric illnesses demanding longterm care. In developing countries, care-giving depends entirely upon the family due to inadequate community based support. ${ }^{5}$ Hence, a shift of attention is required towards caring and supporting the family members.

In a study, that assessed caregiver burden in patient suffering from schizophrenia, the overall burden was found to be moderate and most burden were in areas of finance and family dynamics. ${ }^{6}$ Our study was designed to assess psychological burden in caregivers of patients suffering from Schizophrenia and BPAD.

\section{METHODS}

The study was carried out in Kathmandu Medical College, Sinamangal, Nepal after obtaining an approval from the Ethical Committee and Institution Research Committee of the institute. This study was a cross- sectional, prospective study, carried out from June 2016 to November 2016, in the in-patient unit of department of psychiatry. Written informed consent was obtained from the caregivers and the consenting caregivers meeting our research criteria were enrolled in the study using purposive sampling. The inclusion criteria of the study were: i) Age of the caregiver more than 18 years, ii) caring for more than 2 weeks duration, iii) the caregiver should not have any previously diagnosed psychiatric illness and iv) the caregiver should be present with the patient during maximum period of illness. The diagnosis of BPAD and Schizophrenia was made according to ICD10 .

Self designed, semi- structured performa was used to collect the background information like socio- 
demographic profile of the caregiver, patient related issues such as duration of illness, diagnosis of the patient and self assessment of stress due to their caregiving role. Modified Caregiver Stress Index (MCSI) was used to assess the level of stress related to caregiving. Nepali version of Beck's Depression Inventory (BDI) and Beck's Anxiety Inventory (BAl) were used to assess the presence of depression and anxiety among the caregivers.

Modified Caregiver Strain Index is a 13-item questionnaire, that assesses different domains of caregivers life such as Social, Psychological, Physical, Time, Financial and Employment. It is scored on a scale of 0-2, where 2stands for "Yes", 0 for "No" and 1 for "sometimes". Higher score indicates higher caregiver stress. As there was no cut-off point provided, we arbitrarily choose 13 as the cut-off point, indicating higher level of stress in those who scored more than 13 . The internal reliability coefficient is 0.90 and test- retest reliability coefficient is $0.88 .{ }^{4}$ Beck's Depression Inventory is a 21 item scale used to assess presence and severity of depression. Each item is rated on a four point scale according to the severity of symptoms. Beck's Anxiety Inventory is a 21 item scale used to rate presence and severity of anxiety. Each item is rated on a four point scale. The Nepali version of both BDI and BAI are validated. The sensitivity and specificity of BDI Nepali version is 0.85 and 0.86 with reliability of $0.88,{ }^{7}$ similarly the sensitivity and specificity of BAI Nepali version is 0.91 and 0.89 respectively with reliability of $0.89 .{ }^{8}$ The data was analyzed using SPSS version 20.

\section{RESULTS}

In this study, one hundred caregivers, 50 each of schizophrenia and bipolar affective disorder were enrolled. Out of 100,59 (59\%) were male. The mean age of the caregivers being $43 \pm 12$ years. Eighty- two $(82 \%)$ of the caregivers were married and majority of the caregivers were Spouse $(44 \%)$, followed by parents (30\%). Majority were Hindus (86\%) followed by Buddhist(12\%). Equal numbers of caregivers were from nuclear and joint family (48\%). Forty seven percent were in full time employment, $14 \%$ had part-time jobs and the remaining unemployed. Eighty one percent were taking care of patient whose duration of illness was more than one year. Twenty six percentages said that they were under debt due to illness, among them $30.8 \%$ had debt of over Rs. 50,000.

The participants were asked specifically about being mentally stressed due to caregiving, 71 (71\%) acknowledged being mentally stressed, out of them 61 caregivers (85\%) had higher MCSI score. Overall
72 individuals (72\%) had higher score on MCSI, $25 \%$ of the caregivers had clinical depression, out of which 23 individuals (92\%) had higher MCSI score and 29\% screened positive for anxiety, out of which 28 individuals (96.5\%) had a high MCSI score. Fifty nine caregivers acknowledged that they need professional help to overcome their stress.

The mean score on MCSI was $13.76 \pm 9.981$. The mean MCSI score among caregivers of schizophrenia was $14.00 \pm 5.206$ and among caregivers of BPAD was $13.52 \pm 4.786$; the difference between MCSI score was not found to be statistically significant. The rate of depression among caregivers of schizophrenia and BPAD was found to be $30 \%$ and $20 \%$ respectively and the mean BDI score among caregivers of schizophrenia was $9.64 \pm 9.34$ and among caregivers of BPAD was $8.64 \pm 8.26$; the difference in rate of depression and mean BDI score was not found to be statistically significant. Similarly, $32 \%$ of caregivers of Schizophrenia and $26 \%$ of BPAD were found to have anxiety related problems with mean BAI score being $5.80 \pm 6.38$ and $6.62 \pm 5.84$ respectively. The difference was not found to be statistically significant.

Among the 44 caregivers who were spouse, 36 (81.8\%) had higher level of stress as measured by MCSI and 25 of $30(83.33 \%)$ parent caregiver had higher level of stress. Comparison of different caregiver variables with Modified caregiver strain index score is summarized in Table 1.

Caregiver strain was found to be high and statistically significant with being in debt, longer duration of illness, education level, marital status, subjective feeling of psychological stress and self acknowledgement of need of professional help. Female caregivers were found to have more stress as compared to male; however the difference was not statistically significant. Stress was not found to be associated with family type and employment status of the caregiver.

MCSI score didn't differ significantly with the diagnosis of the patient, meaning caregivers of both the group had similar level of stress. We compared different socio- demographic variables among these two groups of caregivers. There was no significant difference among any variables except for spouse caregiver of Schizophrenic patient experienced greater level of stress than BPAD. It was found that $100 \%$ of the schizophrenic caregiver who were spouse had higher level of psychological stress in comparison to $66.6 \%$ of BPAD caregivers. The other details of comparison of different variables among two groups of caregivers are summarized in Table 2. 


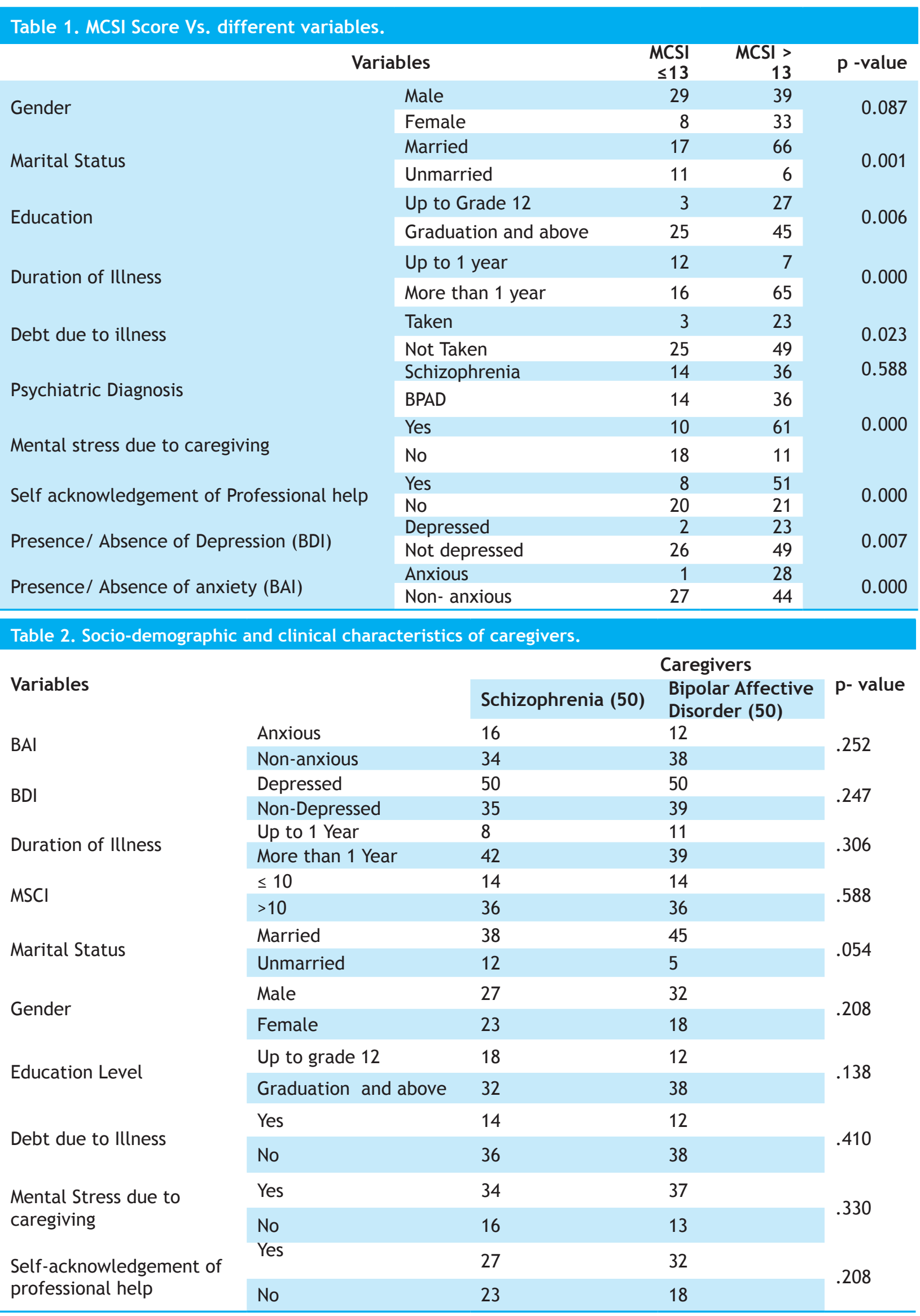




\section{DISCUSSION}

This study found that majority of the caregivers have higher level of stress which was significantly associated with longer duration of illness, being in debt, having lower educational level, married, subjective feeling of psychological stress and self realization of need of medical help. Both group of caregivers experienced similar level of stress but spouse caregiver of Schizophrenic patient had significantly higher level of stress in comparison to BPAD. Moreover, $59 \%$ admitted the need of professional help to overcome the stress. In developing country like ours where community mental health services are not fully functional, family has become an integral part of providing care to patients with psychiatric problems. ${ }^{9}$ However, the mental health status of caregivers has not been given adequate attention. The findings of our study suggest the need to address the mental health problems among caregivers.

In this study, $72 \%$ of the caregivers had higher level of stress. The higher level of stress in our study may be because of the fact that this was a hospital based study and only those caregivers whose patients were in the active phase of the illness and admitted in ward were enrolled in the study. A study conducted in India among psychiatric inpatients, found that the burden was much higher among caregivers of psychiatric patients when compared to chronic medical illness. ${ }^{10} \mathrm{~A}$ study conducted in Pakistan among caregivers of Schizophrenia found $72 \%$ of them were distressed and other studies reporting psychological distress to be as high as $83 \% .{ }^{14-15}$ Due to South Asian cultural similarities, our finding is in consistent with other studies conducted in the same region.

In our study, although female caregivers were found to have higher level of stress than men, this difference was not statistically significant. Studies have shown various socio-demographic factors that may contribute to caregiver stress. Gender of the caregiver is one such factor that has been extensively studied with majority of studies showing female caregivers more likely to experience stress. ${ }^{9,16}$ Study conducted in India, Pakistan and Sri Lanka, however found that gender was not a significant factor contributing to stress. . $^{1,10,15}$

The caregiver stress was found to be significantly high in this study among caregivers who were caring for patients with duration of illness of more than one year. A study conducted among cancer patient yielded similar result. ${ }^{17}$ Our finding is also in accordance to the study conducted in India and Pakistan which revealed higher level of stress in those caregivers who are providing care for longer duration of time ${ }^{10,18}$ The possible explanation to this might be long term care can lead to burnout, financial difficulties, compromises on own priorities which all contributes to development of stress.

This study also found that being in debt was found to be significantly associated with higher level of stress. The possible reason for this might be, patient being an important contributor of the financial support of the family, illness of whose leads to the misbalance of the support system adding to the stress of the family caregiver. Caregiver burden comprises of different factors, financial cost being one of them. ${ }^{11}$ Financial cost incurs both direct and indirect expenses. Some studies have found significant association of financial difficulties with stress. ${ }^{1,19}$

Moreover, we found that married caregivers had higher level of stress than unmarried. The possible reasons for this might be, in our study the majority of caregivers were spouses who were found to have higher level of stress; which could have contributed to the high distress in married caregivers. Another reason might be the added responsibility of children, spouse and other family members contributing to higher level of stress. Another factor that was found to be highly significant with stress was subjective feeling of psychological stress and self acknowledgement of need of medical help. Self dissatisfaction has been associated with higher level of stress. ${ }^{1}$

Furthermore, this study found that lower educational level was associated with higher level of stress. The data regarding education level and stress in published literature is mixed, some studies suggesting higher level of stress in lower education group, some suggesting no association and some suggesting higher level of stress in higher education group..$^{9,15}$ The majority of caregivers were spouse and parents in this study. Our finding is consistent with other studies where parents and spouse were majority of caregivers. ${ }^{20-21}$ Stress was found to be slightly higher among parents when compared to spouses who were providing care $(83.33 \%$ vs $81.81 \%)$ but the difference was not statistically significant. Our findings is in accordance with the findings of other studies that consistently showed stress level to be high among the two group of caregivers. , $22-23^{2}$

The caregivers of both illnesses experienced similar level of stress in this study with $72 \%$ of caregivers of both Schizophrenia and BPAD having higher level of stress. There are limited studies that have compared burden in these two groups. ${ }^{1,11}$ The findings regarding this in published literature are mixed, with some earlier 
studies showing higher level of stress among caregivers of schizophrenia whereas later studies showing the burden to be equal among caregivers of these two diseases. ${ }^{11-13}$ The higher and similar level of stress in both groups may be because of the fact that both schizophrenia and bipolar affective disorder are among the chronic psychiatric illness that demands long term care by family members. Chi-square test of different socio-demographic variables among these two groups of caregivers didn 't show any significant difference except for the relation.

However, stress was found to be relatively higher among spouses of Schizophrenic patient in comparison to that of BPAD. This might be due to the reason that patient with schizophrenia often have delusion of infidelity as a psychopathology along with other delusions and hallucinations, which might have contributed to the extra stress in the spouse caregivers. Our finding is similar to the findings of the study conducted in India, which showed that the demographic variable among the caregivers of Schizophrenia and BPAD caregivers didn't differ significantly. ${ }^{11,13}$

In this study, $25 \%$ of the caregivers had diagnosable depression and $29 \%$ screened positive for anxiety related problems, with both co-morbidities being slightly higher among caregivers of schizophrenia but the difference not being statistically significant. Both depression and anxiety were found to be significantly linked with higher level of stress. Studies have shown association of caregiver strain with depression. ${ }^{1,21}$ The rate of anxiety and depression in our study is less than other studies quoted in this study. ${ }^{1,18}$

\section{CONCLUSIONS}

Stress level was found to be high among caregivers of Schizophrenia and BPAD. Factors like duration of illness, being in debt, lower education level, married, subjective feeling of psychological stress and self realization of need of medical help were found to be significantly associated with higher level of stress.

\section{REFERENCES}

1. Rodrigo C et.al. Caregiver strain and symptoms of depression among principal caregiver of patients with schizophrenia and bipolar affective disorder in Sri Lanka. Int J Ment Health Syst. 2013; 7: 2[Full Text]

2. Ferrario SR, Vitalino P, Zotti AM, Galante E, Fronara R. Alzheimer's disease: usefulness of the Family Strain Questionnaire and the Screen for Caregiver Burden in the study of caregiving- related problems.Int J Geriatr
Psychiatry. 2003;18(12):1110-4.[PubMed]

3. Blood GW, Simpson KC, Dineen M, Raimondi SC: Spouses of individuals with laryngeal cancer: castrain and burden. I Commun Disord. 1994;27(1):19-35.[PubMed]

4. Thronton M, Travis SS. Analysis of the reliability of the modified caregiver strain index. J Gerontol B Psychol Sci Soc Sci. 2003;58(2):S127-32.[PubMed]

5. Ukpong DI, Makanjuola ROA. Emotional distress and strain in relatives of patients with severe mental disorders. West Afr J Med. 2003; 22(2):139-142.[Full Text]

6. Singh PM, Prajapati A. Burden of Schizophrenia on caregivers in Nepal. Nepal Med Coll J. 2013;15(2):140-3. [PubMed]

7. Kohrt BA et.al: Validation of Nepali version of Beck's Depression Inventory. Nepalese Journal of Psychiatry. 2002;2(4): 123-130.[Full Text]

8. Kohrt BA et.al: Validation of Nepali version of Beck's anxiety Inventory. Journal of Institute of Medicine. 2003;25:1-4. [Full Text]

9. Prabhu HRA, Prakash J, Mridula. Care giving in Psychiatry: Evolutionary and dimensional perspectives. Delhi Psychiatry Journal. 2011;14 (1):19-25.[Full Text]

10. Ampalam P, Guntura S, Padma V. A comparative study of caregiver burden in psychiatric illness and chronic medical illness. Indian J Psychiatry. 2012; 54(3): 239-243.[Full Text]

11. Chadda RK, Singh TB, Ganguly KK. Caregiver burden and coping. Soc Psychiatry Psychiatr Epidemiol. 2007 Nov;42(11):923-30.[Pub Med]

12. Chakrabarti S, Raj L, Kulhara P, et al. A comparison of the extent and pattern of family burden in affective disorders and schizophrenia. Indian J Psychiatry. 1995;37(3):10512. [Pub Med]

13. Nehra R, Chakrabarti S, Kulhara P, et al.Caregiver coping in bipolar disorder and schizophrenia: a re-examination. Soc Psychiatry Psychiatr Epidemiol. 2005;40(4):329-36. [Pub Med]

14. MalingoL, Fiorillo A, De Rosa C, Malangone C, Maj M. Family burden in long-term disease: a comparative study in schizophrenia vs. physical disorders. Soc Sci Med. 2005;61(2):313-22.[Pub Med]

15. Shah STH, Sultan SM, Faisal M, Irfan M. Psychological distress among caregivers of patients with Schizophrenia. 
J Ayub Med Coll Abbottabad. 2013;25(3-4):27-30.[Pub Med]

16. Sharma N, Chakrabarti S, Grover G. Gender difference in caregiving among family- caregivers of people with mental illnesses. World J Psychiatry. 2016;6(1): 7-17.[Full Text]

17. Yuosafzai AW, Bhuto N, Ahmer S, Siddiqi MN, Salamat S. Caregivers' stress of cancer patients in a tertiary care hospital. J Postgrad Med Inst. 2008; 22:62-3.[Full Text]

18. Basher S, Naizi RS, Minhas FA, Ali W, Nanjam N. Depression and Anxiety in caregivers of mentally ill patients. J Pak Psychiatr Soc. 2005;2(1):27-33.[Full Text]

19. Lai DWL. Effect of Financial costs on caregiving burden of family caregivers of older adults. SAGE Journals. 2012;114.[Full Text]
20. Grandón P, Janero C, Lemos S. Primary caregivers of schizophrenia outpatients: Burden and predictor variables. Psychiatry Res. 2008;158(3):335-43.[Pub Med]

21. Perlick DA et.al. Rumination, gender and depressive Symptoms associated with caregiving strain in bipolar disorder. ActaPsychiatr Scand. 2012;126: 356-362.[Full Text]

22. Schulz R, Sherwood PR. Physical and Mental Health Effects of Family Caregiving. Am J Nurs. 2008; 108 (9 Suppl):23-27.[Full Text]

23. Penning MJ, Wu Z. Caregiver stress and Mental Health: Impact of Caregiving Relationship and Gender. Gerontologist. 2016;56(6):1102-1113.[Pub Med] 